\title{
Adhesion analysis for niobium nitride thin films deposited by reactive magnetron sputtering
}

\author{
Florina Maria ȘERDEAN ${ }^{1, a}{ }^{*}$, Violeta Valentina MERIE ${ }^{2, b}$, Gavril NEGREA ${ }^{2, c}$, \\ Horea George CRIȘAN ${ }^{1, d}$ \\ ${ }^{1}$ Technical University of Cluj-Napoca, Department of Mechanical Systems Engineering, 103-105 \\ Muncii Avenue, 400641, Cluj-Napoca, Romania \\ 2 Technical University of Cluj-Napoca, Department of Materials Science and Engineering, 103- \\ 105 Muncii Avenue, 400641, Cluj-Napoca, Romania \\ âFlorina.Rusu@omt.utcluj.ro, bVioleta.Merie@stm.utcluj.ro, 'avril.Negrea@ispm.utcluj.ro, \\ dHorea.Crisan@auto.utcluj.ro \\ * corresponding author
}

Keywords: Niobium nitride; Thin films; Atomic force microscope; Surface energy; Adhesion.

Abstract. This paper focuses on determining the adhesion force for four samples of niobium nitride thin films using the atomic force microscope, samples which were deposited by reactive magnetron sputtering on silicon substrates. The main objective is to investigate the influence of the deposition parameters on the adhesion force and other important parameters. Hence, the nitrogen flow rate was varied in order to identify its influence for this type of thin films. Several tests were performed in multiple points of each sample using the spectroscopy in point mode of the atomic force microscope. Using the obtained experimental values and two existing mathematical models for the adhesion force, the surface energy of the niobium nitride thin films was estimated.

\section{Introduction}

Adhesion forces at micro- and nano- scale have been the focus of many researchers' work due to their influence on failure mechanisms in Micro-Electro-Mechanical Systems (MEMS). Due to the intensive growth of MEMS, issues such as reliability and long-term durability have gained importance. At micro-scale such aspects are influenced by the fact that the surface forces become more significant than the body forces. Therefore, it is vital to study characteristics such as roughness or adhesion force, that lead to permanent surface attachment, also known as stiction. Along the years several thin films have been investigated in order to determine the adhesion force and other properties (mechanical, tribological, etc.) [1-2]. Due to their applicability in MEMS industry, thin films still present interest for researchers [3-4] and several methods have been used to deposit niobium nitride ( $\mathrm{NbN}$ ) thin films [5-7].

One of the high-resolution methods that allows to measure the adhesion force is the atomic force microscopy (AFM). The procedure consists in moving the tip of the AFM cantilever close to the sample surface. Due to the existing attraction forces they come in contact (the snap-in phenomenon occurs). The loading phase continues and the cantilever is deflected. During the unloading when the cantilever goes back to its initial position the contact between the tip and the sample is broken (the pull-off phenomenon occurs). The AFM software provides a curve that allows to determine the adhesion force (a representative example is given in Fig. 1). 


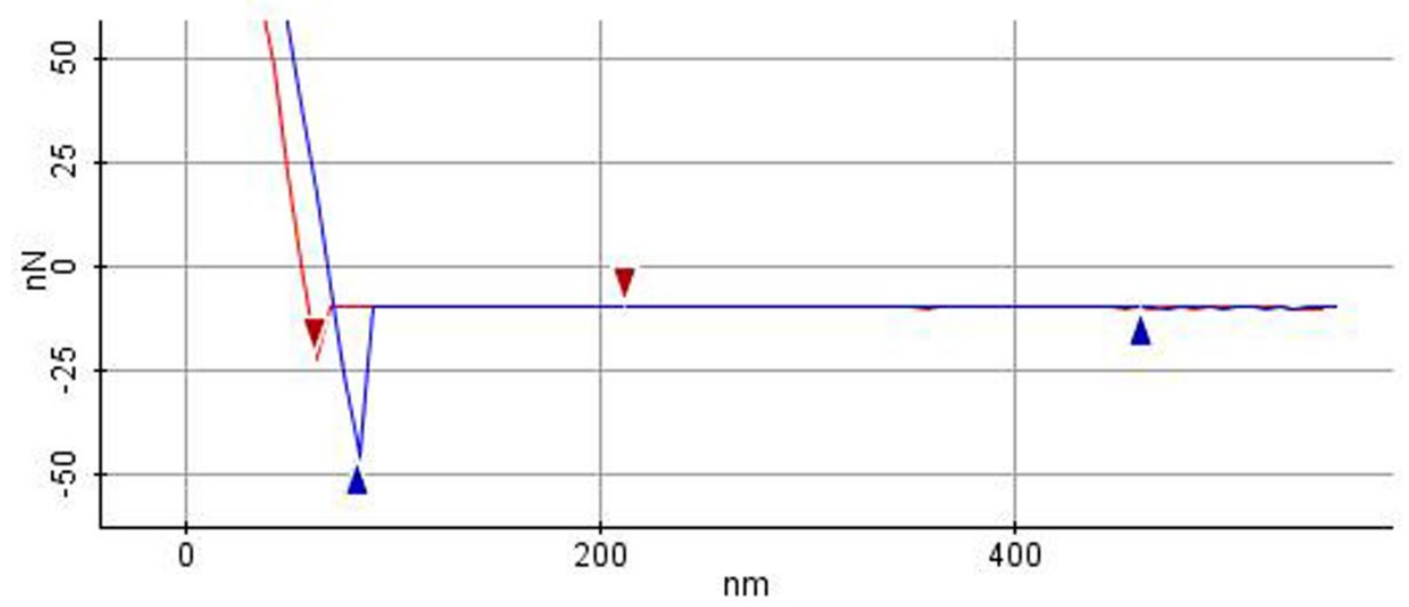

Fig. 1. An example of the force vs. $z$ scan curve for the contact between the AFM tip and the sample surface.

In the open literature there are investigations regarding the influence of fabrication parameters on crystallization, microstructure, and surface composition of NbN thin films [8] and methods such as scratch tests have been used to determine the adhesion [9]. In contrast with the existing research, the present paper encompasses the research regarding the influence of the nitrogen flow rate on the adhesion and other characteristics of $\mathrm{NbN}$ thin films. The experimental investigations were conducted using the spectroscopy in point mode of the AFM.

\section{Materials and experimental procedure}

\section{Materials}

This paper is focused on studying four samples consisting in solid thin films of niobium nitride. They were obtained by depositing one layer of niobium nitride on a silicon Si (100) substrate by reactive magnetron sputtering using a Nb target with a purity of $99.95 \%$.

The deposition was conducted in a high vacuum chamber containing a mixture of argon and nitrogen. Using a constant discharge current $I_{d}$, a constant argon flow rate $Q_{A r}$ and a constant pressure $\mathrm{P}$, all samples were deposited for the same period of time at ambient temperature. The obtained thickness of the deposited thin films was around $0.32 \mu \mathrm{m}$ and it was determined using a JEOL 5600LV electron microscope. As shown in Table 1, the nitrogen flow rate was varied between $0.25 \mathrm{~cm}^{3} / \mathrm{min}$ and $1.75 \mathrm{~cm}^{3} / \mathrm{min}$.

Table 1. Deposition conditions for investigated samples.

\begin{tabular}{|c|c|c|c|c|c|c|}
\hline Thin film & $\begin{array}{c}\mathbf{T} \\
\left({ }^{\circ} \mathrm{C}\right)\end{array}$ & $\begin{array}{l}\text { Time } \\
\text { (min) }\end{array}$ & $\begin{array}{c}P \\
\text { (mtorr) }\end{array}$ & $\begin{array}{c}\mathbf{I}_{d} \\
(\mathbf{m A})\end{array}$ & $\begin{array}{c}\mathbf{Q}_{\mathrm{Ar}} \\
\left(\mathrm{cm}^{3} / \mathrm{mi}\right. \\
\mathrm{n})\end{array}$ & $\begin{array}{c}\mathrm{Q}_{\mathrm{N} 2} \\
\left(\mathrm{~cm}^{3} / \mathrm{mi}\right. \\
\mathrm{n})\end{array}$ \\
\hline Sample 1 & 23 & 20 & 2.2 & 300 & 40 & 0.25 \\
\hline Sample 2 & 23 & 20 & 2.2 & 300 & 40 & 0.75 \\
\hline Sample 3 & 23 & 20 & 2.2 & 300 & 40 & 1.25 \\
\hline Sample 4 & 23 & 20 & 2.2 & 300 & 40 & 1.75 \\
\hline
\end{tabular}


Motivated by the applicability of niobium nitride thin films experimental tests were conducted in order to determine the adhesion force. The software of the AFM used to find the adhesion forces provides several important characteristics that present interest such as roughness together with its characteristic statistics (see for example Fig. 2), the snap-in force or the adhesion energy (see for example Fig. 3 where the results are presented for the third conducted measurement).

\section{Experimental procedure}

The experimental procedure regarding the four thin films at nano-scale was conducted in the Micro \& Nano Systems Laboratory from the Technical University of Cluj-Napoca using a XE 70 AFM. The investigations were performed at a temperature of $23{ }^{\circ} \mathrm{C}$, a relative humidity of $35 \%$ and a scanning frequency of $0.75 \mathrm{~Hz}$. The cantilever used for the tests is a NSC35C cantilever which, according to the specifications provided by the manufacturer has a width of $35 \mu \mathrm{m}$, a length of $130 \mu \mathrm{m}$, a thickness of $2 \mu \mathrm{m}$, a force constant of $5.4 \mathrm{~N} / \mathrm{m}$ and a resonance frequency of $150 \mathrm{kHz}$. The tip radius of the cantilever is smaller than $20 \mathrm{~nm}$. The XEI Image Processing Tool for SPM (Scanning Probe Microscopy) Data was used for data interpretation.

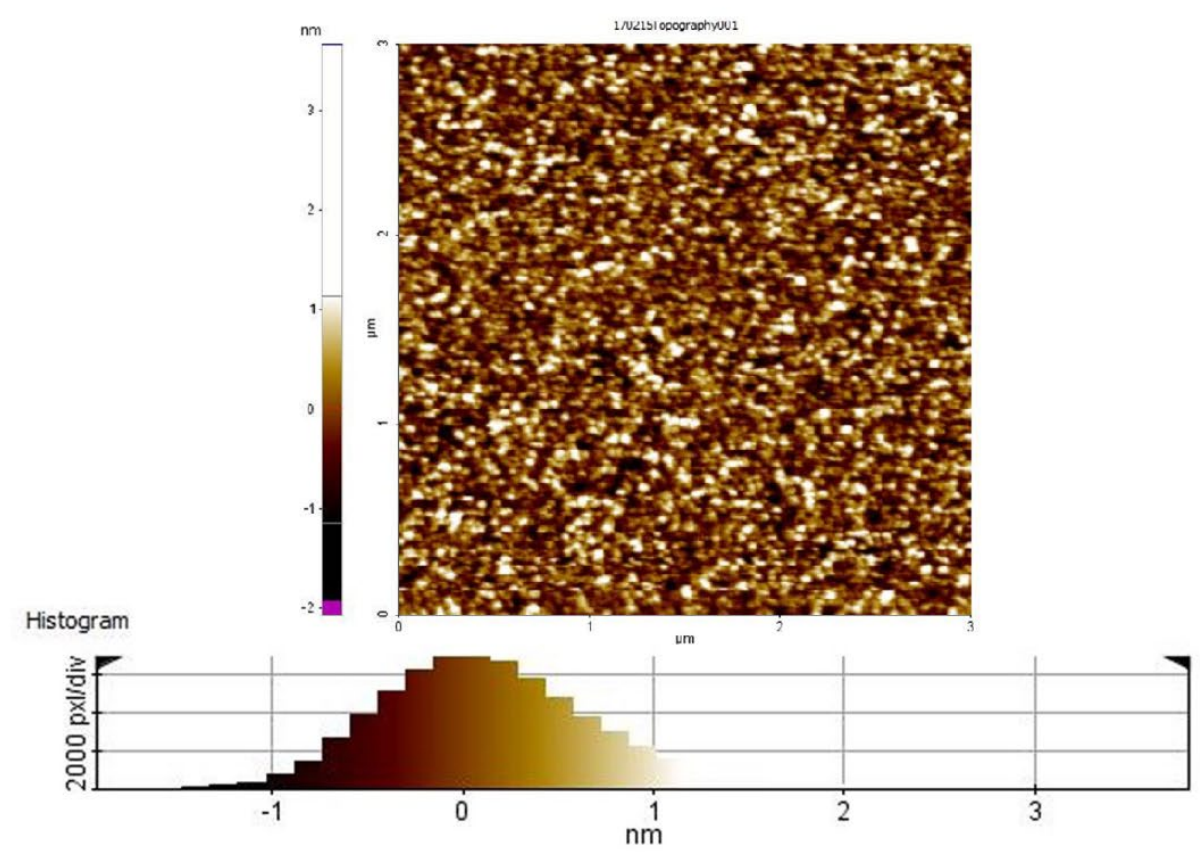

\begin{tabular}{|c|c|c|c|c|c|c|c|c|c|c|}
\hline Region & $\operatorname{Min}(n m)$ & $\operatorname{Max}(\mathrm{nm})$ & $\operatorname{Mid}(\mathrm{nm})$ & Mean $(\mathrm{nm})$ & $\operatorname{Rpv}(\mathrm{nm})$ & $\mathrm{Rq}(\mathrm{nm})$ & $\mathrm{Ra}(\mathrm{nm})$ & $\mathrm{Rz}(\mathrm{nm})$ & Rsk & Rku \\
\hline 필 Whole & -1.913 & 3.661 & 0.874 & 0.000 & 5.574 & 0.580 & 0.456 & 4.992 & -0.512 & 3.579 \\
\hline
\end{tabular}

Fig. 2. The surface of Sample 3 and the statistical parameters provided for its roughness by the AFM software.

The technique used to determine the adhesion force (the pull-off force) between the AFM tip and the NbN thin films, as well as other parameters of interest such as the snap-in force and adhesion energy is the spectroscopy in point mode of the AFM. This technique returns as output together with values for different parameters, some AFM experimental curves similar to the one presented in Fig. 3 which present both the loading and the unloading phases of the procedure. The values of the adhesion forces between the AFM tip and each of the four NbN thin films were 
obtained based on these curves and determined automatically by the software (see in Fig. 3 the value for the pull-off force). The measurements of the adhesion force were conducted in 5 random points for each sample and the average value was computed. The points were chosen by studying the 3D image of each sample (see for example Fig. 4) so that any defects are avoided. This procedure was repeated for roughness, snap-in force and adhesion energy.

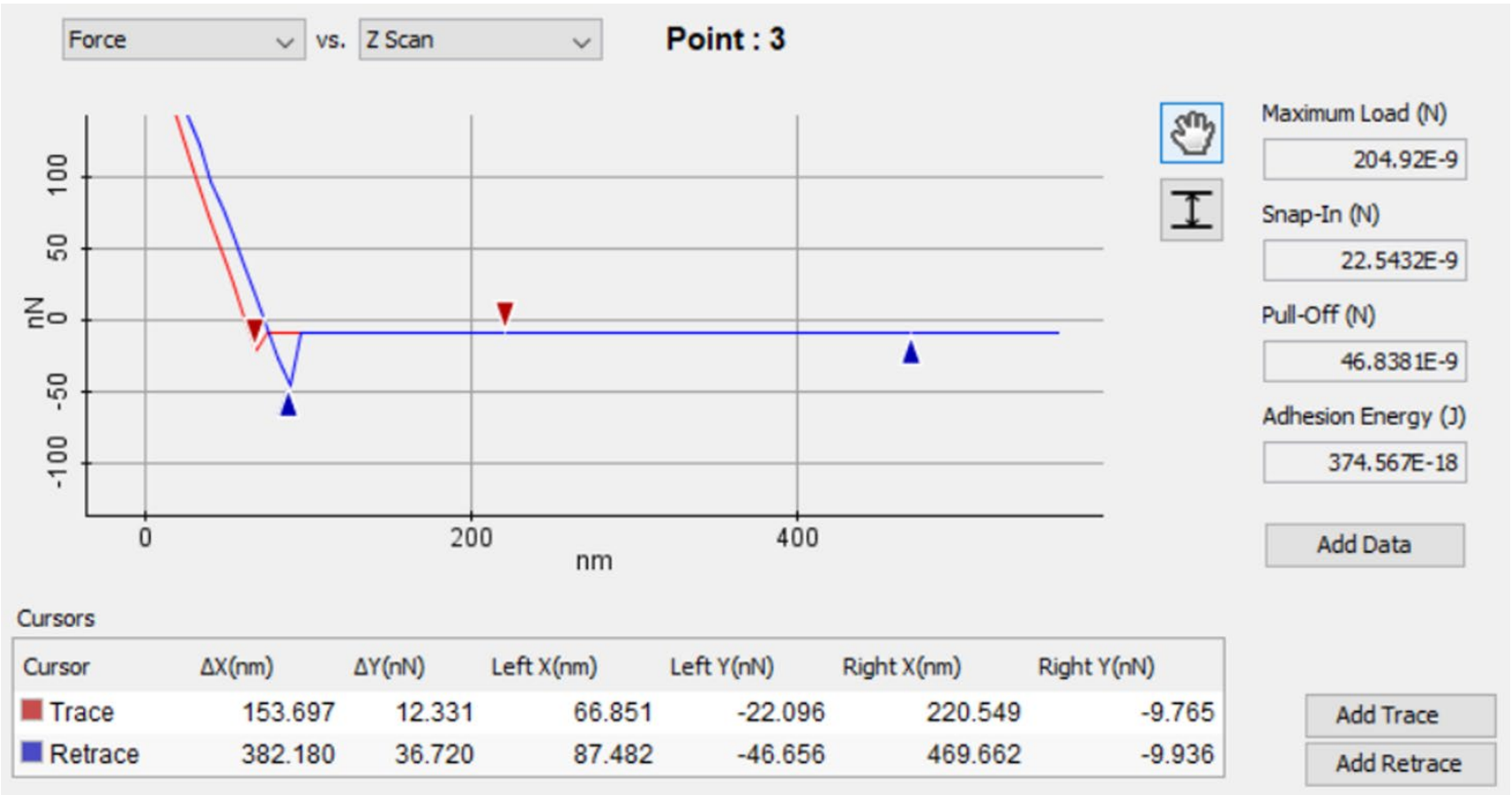

Fig. 3. The force vs. $\mathrm{z}$ scan curve together with the charactristics provided by the AFM software for an experimental test conducted on Sample 3.

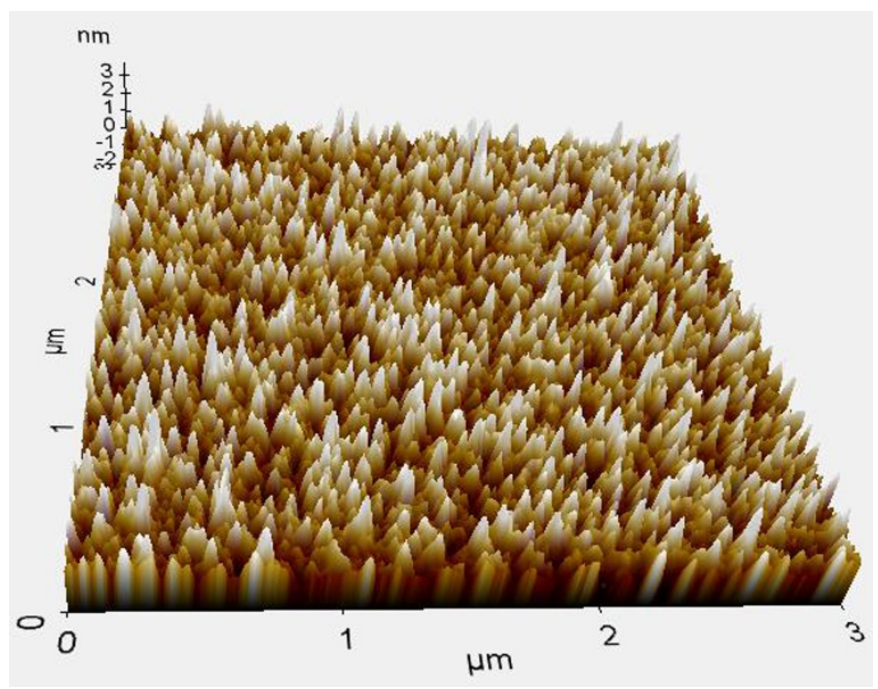

Fig. 4. 3D image of the third sample. 


\section{Theoretical models}

One of the main factors that influence the adhesion is the type of contact between the surfaces. This contact has been modelled in different ways along the years of research in order to accurately estimate the adhesion force. Two of the most known and used models are the JKR (JohnsonKendall-Roberts) model and the DMT (Derjaguin-Müller-Toporov) model. These models were established due to the need to remove the differences between the values when applying experimental tests and when applying the Hertz theory for low loads. In both models the adhesion force is expressed as a function of the surface energy [10]:

$$
\begin{aligned}
& F_{a d}^{J K R}=\frac{3}{2} \pi \gamma R \\
& F_{a d}^{D M T}=2 \pi \gamma R
\end{aligned}
$$

where $\gamma$ is the surface energy and $R$ is the radius of the AFM tip. These models are used for the contact between the AFM tip and a surface with low roughness.

\section{Results and discussions}

The main objective of this paper is to study the influence of the nitrogen flow rate on the adhesion force and other important characteristics. Therefore, for each sample the experimental measurements were repeated five times and the average of the obtained values is presented in Table 2 for roughness, attraction forces and adhesion energy, respectively. For the adhesion energy all five measurements together with the average and standard deviation are included in Table 3.

Table 2. Average experimental values for all investigated samples.

\begin{tabular}{|c|c|c|c|}
\hline Thin film & $\begin{array}{c}\text { Roughness } \\
\mathbf{( n m )}\end{array}$ & $\begin{array}{c}\text { Attraction } \\
\text { forces (nN) }\end{array}$ & $\begin{array}{c}\text { Adhesion energy } \\
\left(\mathbf{J} \cdot \mathbf{1 0}^{-\mathbf{1 8}} \mathbf{)}\right.\end{array}$ \\
\hline Sample 1 & 0.531 & 33.25 & 630 \\
\hline Sample 2 & 0.679 & 12.80 & 838 \\
\hline Sample 3 & 0.456 & 21.62 & 362 \\
\hline Sample 4 & 1.252 & 14.42 & 223 \\
\hline
\end{tabular}

Table 3. Experimental values for the adehsion force.

\begin{tabular}{|c|c|c|c|c|c|c|c|}
\hline Mhin film & $\mathbf{1}$ & $\mathbf{2}$ & $\mathbf{3}$ & $\mathbf{4}$ & $\mathbf{5}$ & $\begin{array}{c}\text { Average } \\
(\mathbf{n m})\end{array}$ & $\begin{array}{c}\text { Standard } \\
\text { Deviation } \\
\mathbf{( n m})\end{array}$ \\
\hline Sample 1 & 75.61 & 86.21 & 89.53 & 69.94 & 77.92 & 79.84 & 7.97 \\
\hline Sample 2 & 57.34 & 62.38 & 68.41 & 76.95 & 70.14 & 67.04 & 7.51 \\
\hline Sample 3 & 40.13 & 46.84 & 43.17 & 49.26 & 48.97 & 45.67 & 3.94 \\
\hline Sample 4 & 40.13 & 37.66 & 35.45 & 29.05 & 34.15 & 35.29 & 4.16 \\
\hline
\end{tabular}


As it can be seen the first sample has the highest value for the adhesion force. The adhesion force is strongly influenced by the surface free energy which is specific for each sample, therefore influenced by the nitrogen flow rate used at deposition. We believe that a decrease of the surface free energy of the thin films occurs with the increase of the nitrogen flow rate. Hence, it can be noted that using the same deposition parameters the adhesion force decreases up to almost 56\% with the increase of the nitrogen flow rate.

By analyzing the data presented in the same table it can be seen that the other studied parameters do not follow a strictly increasing or strictly decreasing trend. The roughness values first increase, then a drop can be noted for the third sample and in the end for the sample obtained at the highest nitrogen flow rate, the highest average value for the roughness was obtained.

The attraction forces (snap-in forces) for the second sample are lower than for the first one, then the values increase to a higher value for the third sample and then decrease again for the fourth. The adhesion energy is characterized by an increase in values when comparing the first two samples, followed by a dramatic decrease for the last two samples.

Table 4. Surface energy for all investigated samples.

\begin{tabular}{|c|c|c|c|c|}
\hline$\gamma_{\left(\mathbf{J} / \mathbf{m}^{2}\right)}$ & Sample 1 & Sample 2 & Sample 3 & Sample 4 \\
\hline Minimum value & 0.706 & 0.593 & 0.404 & 0.312 \\
\hline Maximum value & 0.941 & 0.790 & 0.538 & 0.416 \\
\hline
\end{tabular}

Using the average values obtained experimentally for the adhesion force and the two mathematical models presented above, the surface energy was estimated and the computed values are presented in Table 4.

\section{Summary}

The elaboration using in general different deposition parameters such as in this case the nitrogen flow rate determines different material properties possibly due to the preferential orientation of the atoms planes. The results of this study concerning the influence of the nitrogen flow rate on the properties of $\mathrm{NbN}$ thin films deposited by reactive magnetron sputtering on silicon substrate have shown it influences all studied parameters. The obtained results allowed even to quantify the degree of influence on the adhesion force.

The conducted experimental work has not indicated a certain direct correlation such as direct proportionality or inverse proportionality or even the same or inverse trend between the nitrogen flow rate and the other investigated parameters besides the adhesion force. However, the results show that the studied parameters are influenced by the increase of nitrogen flow rate when the other deposition parameters were kept constant. The roughness and the attractive forces have an inverse trend variation. Moreover, the tests proved a direct correlation for the case of the adhesion force values which decreased with the nitrogen flow rate increase.

The XE 70 AFM used for the experimental investigations and the XEI Image Processing Tool for SPM data used for the interpretation of the data allowed to determine the roughness of each of the four samples. Due to the low roughness values mathematical models suitable for the contact between a sphere and a plane were used to model the contact between the AFM tip and the sample surfaces. Based on these models the surface energy of the NbN thin films was computed. The obtained values indicate that it can vary up to three times depending on the nitrogen flow rate. 


\section{Acknowledgement}

The results presented in this paper were obtained with the support of the Technical University of Cluj-Napoca through the research Contract no. 2003/12.07.2017, Internal Competition CICDI2017. The authors would like to thank Professor Marius Pustan and Professor Corina Bîrleanu for easing the access to the atomic force microscope.

\section{References}

[1] Z. Stanimirović and I. Stanimirovic, Mechanical properties of MEMS materials, Micro Electronic and Mechanical Systems, book edited by: Kenichi Takahata, p. 572, December 2009, INTECH, Croatia.

[2] A. D. Pogrebnjak, O. V. Bondar, G. Abadias, V. Ivashchenko, O. V. Sobol, S. Jurga and E. Coy, Structural and mechanical properties of NbN and Nb-Si-N films: Experiment and molecular dynamics simulations, Ceram. Int., $42 \quad$ (2016) 11743-11756. https://doi.org/10.1016/j.ceramint.2016.04.095

[3] Y. Lei et al., Fabrication of niobium titanium nitride thin films with high superconducting transition temperatures and short penetration lengths, IEEE Trans. Appl. Supercond., 15 (2005) 44-48. https://doi.org/10.1109/TASC.2005.844126

[4] C.-L. Huang et al., Characteristics of reactively sputtered niobium nitride thin films as diffusion barriers for $\mathrm{Cu}$ metallization, Electron. Mater. Lett., 9 (2013) 593-597. https://doi.org/10.1007/s13391-012-2173-0

[5] M. Ziegler et al., Superconducting niobium nitride thin films deposited by metal organic plasma-enhanced atomic layer deposition, Supercond. Sci. Technol., 26 (2013) 025008-5. https://doi.org/10.1088/0953-2048/26/2/025008

[6] Y. Ufuktepe et al., Superconducting niobium nitride thin films by reactive pulsed laser deposition, Thin Solid Films, 545 (2013) 601-607. https://doi.org/10.1016/j.tsf.2013.08.051

[7] F. Mercier et al., Niobium nitride thin films deposited by high temperature chemical vapor

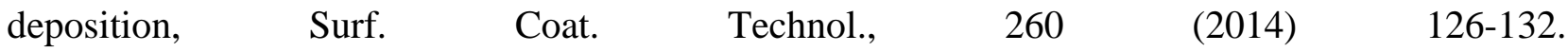
https://doi.org/10.1016/j.surfcoat.2014.08.084

[8] J.E. Alfonso, J. Buitrago, J. Torres et al., Influence of fabrication parameters on crystallization, microstructure, and surface composition of $\mathrm{NbN}$ thin films deposited by rf magnetron sputtering, J. Mater. Sci., 45 (2010) 5528-5533. https://doi.org/10.1007/s10853010-4612-3

[9] Y. J. Totik, Investigation of the adhesion of $\mathrm{NbN}$ coatings deposited by pulsed dc reactive magnetron sputtering using scratch tests, Coat. Technol. Res., 7 (2010) 485-492. https://doi.org/10.1007/s11998-009-9200-6

[10] D.S. Grierson, E.E. Flater, R.W. Carpick, Accounting for the JKR-DMT transition in adhesion and friction measurements with atomic force microscopy, J. Adhes. Sci. Technol., 19 (2005) 291-311. https://doi.org/10.1163/1568561054352685 